\title{
Supporting Aphasics for Capturing, Organizing and Sharing Personal Experiences*
}

\author{
Abdullah Al Mahmud \\ Department of Industrial Design, Eindhoven University of Technology \\ P.O.Box 513, $5600 \mathrm{MB}$ Eindhoven, The Netherlands \\ \{a.al-mahmud, j.b.o.s.martens\} @tue.nl
}

\begin{abstract}
When a person, due to brain injury or another disease, suffers in his or her ability to speak, it becomes inherently cumbersome to share needs, emotions, and experiences through personal stories and social interaction. This paper describes the aim and progress of the author's dissertation, which focuses on designing a support system to share daily experiences for people suffering from expressive aphasia.
\end{abstract}

Keywords: Aphasia, Sharing experiences, Contextual interview.

\section{Introduction}

Much of our social life consists of sharing daily stories with other people. However, sharing personal stories can be extremely difficult for people with limited verbal ability, such as those suffering from expressive aphasia. As a result, aphasia often leads to increased social isolation and possibly to depression. Enabling aphasics to share their daily experiences will help them to become more socially active and to re-engage with their preferred life style. Aphasia is an acquired communication disorder that is caused by brain injury or trauma. Aphasia affects language comprehension and generation [4], such that people's ability to express themselves verbally suffers. Augmentative and Alternative Communication (AAC) devices such as TouchSpeak [7] are widely used in aphasia therapy as well as during the post-therapy period but there are various limitations of AAC. They contain isolated symbols whose meanings have to be learned and aphasics need to retrieve phrases or words to construct meaning in order to support very simple stories. Therefore, the way these activities are designed end up posing a cognitive challenge for the users. Moreover, AAC devices support needs-based interaction for functional communication. They lack the support for sharing experiences based on real life events, which are crucial aspects to social interaction.

Photographs have several functions such as preserving memories about the past, narrating and organizing everyday experiences, and communicating and building social relations [3]. Photography has been proposed within 'Aphasia Talks' [6] as a way of facilitating self-expression in aphasics for reintegration, improving socialization and allowing recreation. The usefulness of digital photographs within

*Advisor: Jean-Bernard Martens. 
conventional storytelling has been reported (see e.g iTell [5]). The challenge or issue of how people with special needs can be empowered to use photos to share experience, does not yet seem to have been explored in depth. A study has been reported to help aphasics in building story using photos [2]. However, there were certain limitations of the prototype such as the editing of the story had to be done at the time of capture and there was no support for organizing the captured photos. Moreover, the story-building task was limited just by adding a simple emoticon and sound and lacking the order and comprehensibility of the captured materials. Therefore, our objective is to design a supporting system for capturing and sharing personal experiences via photos considering the limitations described above.

The main research question of this $\mathrm{PhD}$ thesis is to investigate whether and how capturing everyday activities that assist in storytelling can help to reduce the social isolation and passive position of aphasics. We specifically focus on aphasics who have been discharged from the rehabilitation center and who need to continue their life with a serious disability. The main reason for this is that in earlier phases of diagnosis and treatment, therapists and patients tend to concentrate on training whatever speech abilities that remain.

\section{Methodology and Conducted Research}

Since aphasics cannot express their thoughts verbally, it is not easy to involve them in the design process. Existing research focused on different methods such as participatory design by involving domain experts in a user centered manner etc [1]. We involved speech therapists in the design process since they are able to judge the capabilities of aphasics. To know more about how aphasics and how their partners are involved in the communication process we visited one aphasic and his partner. Moreover, we also explored whether and how photos can be used to tell past experience. The main findings were a. photos can be used for aphasics to recall past experience b. Personally relevant photos are needed to narrate an experience c. providing personally relevant cues are helpful such as 'who', 'what', to make the process of retelling easier.

We realized that it was not easy to engage aphasics and elicit design requirements. Therefore, we have adopted a different methodology. We conducted an experiment with two non-aphasic persons and they were asked to explain how they would capture and share their personal experiences in an unfamiliar language. This would mirror the task that aphasics would need to carry out if they would narrate their story using photos. We argue that involving non-aphasics in the early design phase will give us better insight to understand the problem of expressive aphasics. Participant was told to take photos of events and express that photo event(s) in an unfamiliar language. The main objective was to find out how people construct and share narratives from photos to others given that they did know a particular language, similar to the condition of aphasics. The main findings are a. not many pictures are needed for communicating key everyday experience b. people miss joining words, phrases to establish a connection between photos while telling in other language $\mathrm{c}$. people prefer to narrate their experience by making clusters based on activity and time. The findings are used to enrich the first conceptual prototype, which is described below. 


\section{Proposed Solution}

The design we propose will help aphasics to capture everyday experiences and later to share it with significant others in a face-to-face setting. There are two aspects of the proposed design. First is the capturing of everyday experiences. It became apparent that a traditional camera is not suitable for aphasics to capture daily experiences [6]. Therefore, we propose to build a suitable capturing device such as using an off the shelf sunglass camera to capture picture, sound and video (see Fig 1). The captured device will be operated by a remote controller and that can easily be handled by only one hand. Later, these captured materials can easily be transferred to a laptop or PC for reviewing with the help of our appropriate sharing software application. A suitable docking station will be designed for transferring the captured materials easily.

Computing technology can provide support to construct a particular experience from the captured materials. The support can be in the form of help in organizing and editing the captured materials and guiding user to recall in constructing experiences. As a first step in designing the first prototype, we have created an application that could help aphasics to organize and share personal experiences (see Fig 1). The application facilitates aphasics to organize their collected photos according to time and date. Moreover, photos can be organized according to different clusters such as 'who', 'where' 'what' and a story can be defined in terms of these principles. To help in building a narrative to share experience a template is provided. The template has the option to fill appropriate pictures according to the attributes of who, where and what. There is an option for adding personal media such as audio, text, inbuilt icons in the picture to augment its meaning. Being able to respond to questions is obviously, what

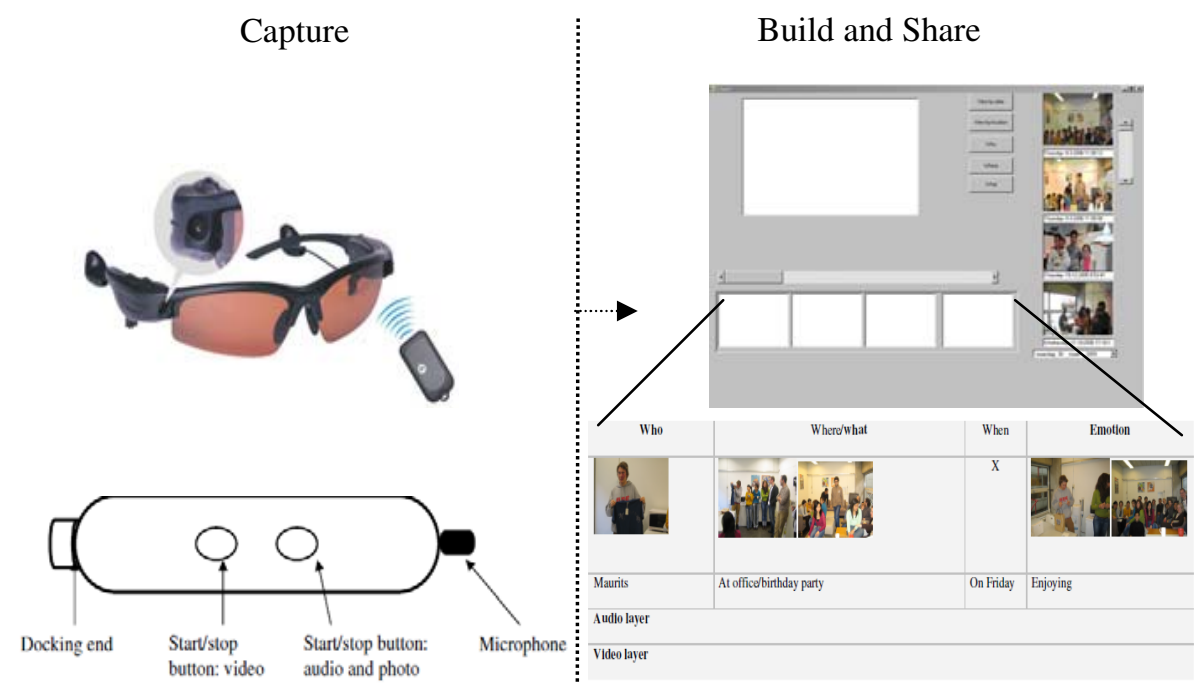

Fig. 1. The capturing device with external controller (left), the application for building and expressing narrative of experiences (right) 
makes the difference between simply telling a story and using storytelling as a means for social interaction. This aspect therefore needs to be carefully developed and tested in interaction with the end user.

\section{Research Plan}

First, we will evaluate the suitability of the capturing device. The tool will be evaluated in a longitudinal study to investigate how aphasics use the device in their daily life. Moreover, we want to compare how aphasics and non-aphasics use the device and can communicate by using it effectively. We will incorporate the findings while evaluating it iteratively in to the final design. The next step is to evaluate the application for constructing and sharing daily experiences. It is equally important to evaluate how the narrative template that we proposed helps aphasics to reconstruct their experiences. Moreover, several design cycles with proxies are planned, also because we want to explore how the use of an experience sharing application evolves over time as users become more proficient with it.

Expected Contributions. Prior research has not explored how capturing and sharing could improve social interaction and confirming social affiliations for aphasics. We expect to show experimentally how the passive position of aphasics can be alleviated by our proposed solution. The method that we deploy will also contribute for designing assistive technologies for aphasics. The research and design outlined in this paper, although aimed at people with (expressive) aphasia, may be relevant for other user groups, such as people suffering from dementia or Alzheimer disease.

Acknowledgement. I would like to thank my supervisor Prof. Jean-Bernard Martens for his continuous support. Thanks also go to speech therapists, the person with aphasia and his partner for their cooperation.

\section{References}

1. Allen, M., McGrenere, J., Purves, B.: The Field Evaluation of a Mobile Digital Image Communication Application Designed for People with Aphasia. ACM Trans. Access. 1(1), $1-26(2008)$

2. Daemen, E., Dadlani, P., Du, J., Li, Y., Erik-Paker, P., Martens, J.B., de Ruyter, B.: Designing a free style, indirect, and interactive storytelling application for people with aphasia. In: Baranauskas, C., Palanque, P., Abascal, J., Barbosa, S.D.J. (eds.) INTERACT 2007. LNCS, vol. 4662, pp. 221-234. Springer, Heidelberg (2007)

3. Harrison, B.: Photographic visions and narrative inquiry. J. Narrative Inquiry 12(1), 87-111 (2002)

4. Hillis, A.E.: Aphasia: Progress in the last quarter of a century. Neurology 69, 200-213 (2007)

5. Landry, B.M., Guzdial, M.: iTell: supporting retrospective storytelling with digital photos. In: Proc. DIS 2006, pp. 160-168 (2006)

6. Levin, T., Scott, B., Borders, B., Hart, K., Lee, J., Decanini, A.: Aphasia Talks: photography as a means of communication, self-expression, and empowerment in persons with aphasia. J. Topics in stroke rehabilitation 14(1), 72-84 (2007)

7. TouchSpeak, http://www.touchspeak.co.uk/ 